Article

\title{
Phylogeographical Structure of Liquidambar formosana Hance Revealed by Chloroplast Phylogeography and Species Distribution Models
}

\author{
Rongxi Sun ${ }^{1,2,+} \mathbb{D}$, Furong Lin ${ }^{1,+}$, Ping Huang ${ }^{1}$, Xuemin Ye ${ }^{2}$, Jiuxin Lai ${ }^{1}$ and Yongqi Zheng ${ }^{1, * \mathbb{D}}$ \\ 1 State Key Laboratory of Tree Genetics and Breeding, Key Laboratory of Silviculture of the State Forestry \\ Administration, Research Institute of Forestry, Chinese Academy of Forestry, Beijing 100091, China; \\ rongxisun@163.com (R.S.); linfr888@163.com (F.L.); pippin09@163.com (P.H.); jackolai@126.com (J.L.) \\ 2 Jiangxi Provincial Key Laboratory of Silviculture, College of Forestry, Jiangxi Agricultural University, \\ Nanchang 330045, China; yexuemin0827@163.com \\ * Correspondence: zyq8565@126.com; Tel.: +86-10-6288-8565 \\ + These authors contributed equally to this work.
}

Received: 2 September 2019; Accepted: 29 September 2019; Published: 1 October 2019

\begin{abstract}
To understand the origin and evolutionary history, and the geographical and historical causes for the formation of the current distribution pattern of Lquidambar formosana Hance, we investigated the phylogeography by using chloroplasts DNA (cpDNA) non-coding sequences and species distribution models (SDM). Four cpDNA intergenic spacer regions were amplified and sequenced for 251 individuals from 25 populations covering most of its geographical range in China. A total of 20 haplotypes were recovered. The species had a high level of chloroplast genetic variation $(\mathrm{Ht}=0.909 \pm 0.0192)$ and a significant phylogeographical structure (genetic differentiation takes into account distances among haplotypes (Nst) $=0.730>$ population differentiation that does not consider distances among haplotypes (Gst) $=0.645 ; p<0.05$ ), whereas the genetic variation within populations (Hs $=0.323 \pm 0.0553$ ) was low. The variation of haplotype mainly occurred among populations (genetic differentiation coefficient $(\mathrm{Fst})=0.73012$ ). The low genetic diversity within populations may be attributed to the restricted gene flow $(\mathrm{Nm}=0.18)$. The time of the most recent common ancestor for clade V mostly distributed in Southwestern China, Central China, Qinling and Dabieshan mountains was $10.30 \mathrm{Ma}$ (95\% Highest posterior density (HPD): 9.74-15.28) dating back to the middle Miocene, which revealed the genetic structure of L. formosana was of ancient origin. These results indicated that dramatic changes since the Miocene may have driven the ancestors of L. formosana to retreat from the high latitudes of the Northern Hemisphere to subtropical China in which the establishment and initial intensification of the Asian monsoon provided conditions for their ecological requirements. This scenario was confirmed by the fossil record. SDM results indicated there were no contraction-expansion dynamics, and there was a stable range since the last interglacial period (LIG, 130 kya). Compared with the population expansion detected by Fu's Fs value and the mismatch distribution, we speculated the expansion time may happen before the interglacial period. Evidence supporting L. formosana was the ancient origin and table range since the last interglacial period.
\end{abstract}

Keywords: Liquidambar formosana; altingiaceae; China; neogene relict plant; cpDNA; species distribution models; range stable; ancient origin

\section{Introduction}

Asia's climate has experienced dramatic changes since the Miocene and the global climate cooling (17-15 Ma) after the Miocene [1,2]. The dramatic changes included intensification of East Asian 
monsoons (20-15 Ma) [3], and aridification in Central Asia (22 Ma) [4]. The uplift of the Qinghai-Tibetan Plateau (QTP) began with the collision of the Indian Plate with Eurasia Plate (55-50 Ma) [5]. Further uplift of the QTP caused intensifications of the East Asian summer and winter monsoons (15 Ma [6], 10-8 Ma, and 3.6-2.6 Ma [7]). In the Pleistocene, the monsoon system interacting with glacial-interglacial cycles, produced a more variable monsoon climate [7].

Relatively little attention has been focused on the intraspecific level since the Miocene because the divergence time within species rarely trace that far back [8]. Nonetheless, a few studies on the evolutionary histories of relict plants traced back to the Middle and Early Miocene, a period that had experienced dramatic climate and topographic changes in Asia [1,2,7,9]. Subtropical China is the most abundant region of Neogene relict plants and glacial refugia of plants in the Quaternary, as well as an important source of post-glacial plant dispersion [10]. Compared to other temperate/subtropical regions, such as southern Europe and the southeastern United States where genetic structure is often of recent origin and related to refugia dynamics, the ancient origin of the relict plant in subtropical China may have experienced less extreme demographic fluctuations [9]. Currently, phylogeographical studies of subtropical China are mainly focused on rare and endangered plants, such as Sargentodoxa cuneate (Oliv.) [11], Davidia involucrate Baill. [12], Cercidiphyllum japonicum. [13]. Compared with these endangered plants, widespread species which had larger distribution areas provide a useful model to comprehensively understand the evolutionary patterns and range dynamics.

Liquidambar formosana Hance is a Neogene relict plant of the genus Liquidambar from the monotypic family Altingiaceae are mainly distributed in subtropical China and has a long evolutionary history [14,15]. Genus Liquidambar had flourished well in a wide region covering East Asia, Central Asia, Asia Minor, America, and Central Europe during the Miocene, and then disappeared in Europe and Northwest America in the Pleistocene as a result of extensive glaciations [16,17]. Although now restricted in the south of China, L. formosana has a long fossil record of pollen (about $70 \mathrm{Ma}$ ) in the northeast of China. L. formosana is a pioneer and fast-growing deciduous tree species. Its natural distribution across Southern China, north from Qinling and Dabieshan Mountains, west to Sichuan and Guizhou, south to Hainan, and east to Taiwan [18]. This species occurs across a broad elevational range (100 m-1600 m) and environments. Due to its geographical distribution and ecological amplitude, L. formosana is highly suitable for investigating the effects of past geological and climatic changes on the population dynamics.

A previous study based on four chloroplast markers and two nuclear makers indicated that L. formosana and Liquidambar acalycina may have multiple glacial refugia in East, South, and Southwest China, but the phylogeographic structure was weak [19]. Population genetic analysis based on simple sequence repeat (SSR)and inter simple sequence repeat (ISSR) markers showed a moderate genetic diversity and moderate level of population differentiation $[18,20]$. However, the information on the evolutionary history of the species was limited by small sample size and a narrow sampling range.

We conducted phylogeographical analyses of L. formosana using four chloroplasts DNA intergenic spacer regions ( $p s b D$-trnT, psbJ-pet A, trnL-trnF, and $n d h F-r p l 32$ ) which has a maternal inheritance, a slow evolution rate [21,22], and the lack of recombination as in most angiosperms [23]. Meanwhile, we used ensemble species distribution models for inferring past potential distribution at different periods based on the existing data of geographical distribution and climate. We address the following questions: (1) Infer the intraspecific evolutionary history to test if the genetic structure was of recent or ancient origin. (2) Infer the range dynamics to test whether there were refugial dynamics or stable range through time. Studies on the phylogeography of L. formosana are of great significance to understand the origin and evolutionary patterns, and to explore the geographical and historical causes for the formation of the current distribution pattern. 


\section{Materials and Methods}

\subsection{Plant Materials and DNA Extraction}

A total of 251 individual trees of L. formosana were collected from 25 populations covering most of its geographical range in China (Figure 1, Table 1). Fresh young leaves were collected from 10 to 11 sample trees, with a minimum distance of $100 \mathrm{~m}$ apart from each other. One sample of Liquidambar styraciflua was used in the phylogenetic analysis as outgroup. The leaf samples were then dried using silica gel for DNA extraction. Total genomic DNA was extracted using the Plant Genomic DNA Kit (TIANGEN, Beijing, China). The DNA quality and concentration were measured by electrophoresis on $0.8 \%$ agarose gel and Microplate Spectrophotometer (Molecular Device, Sunnyvale, CA, USA) respectively. The DNA samples were diluted to $50 \mathrm{ng} / \mu \mathrm{L}$ for a later experiment.

Table 1. Sampling information of Liquidambar formosana.

\begin{tabular}{cccccc}
\hline $\begin{array}{c}\text { Population } \\
\text { Code }\end{array}$ & Sampling Location & $\begin{array}{c}\text { Number of } \\
\text { Sample Trees }\end{array}$ & Longitude (E) & Latitude (N) & Altitude (m) \\
\hline BWL & Bawangling, Hainan & 10 & $108^{\circ} 48^{\prime}$ & $18^{\circ} 30^{\prime}$ & 655 \\
PX & Pingxiang, Guangxi & 10 & $106^{\circ} 50^{\prime}$ & $22^{\circ} 10^{\prime}$ & 420 \\
TR & Tongren, Guizhou & 10 & $108^{\circ} 56^{\prime}$ & $27^{\circ} 47^{\prime}$ & 400 \\
XY & Xingyi, Guizhou & 10 & $104^{\circ} 57^{\prime}$ & $25^{\circ} 04^{\prime}$ & 1100 \\
HSAH & Huangshan, Anhui & 10 & $117^{\circ} 36^{\prime}$ & $30^{\circ} 08^{\prime}$ & 150 \\
KX & Kangxian, Gansu & 10 & $105^{\circ} 46^{\prime}$ & $33^{\circ} 09^{\prime}$ & 1020 \\
LY & Lueyang, Shaanxi & 10 & $105^{\circ} 50^{\prime}$ & $32^{\circ} 20^{\prime}$ & 700 \\
TG & Tonggu, Jiangxi & 10 & $114^{\circ} 19^{\prime}$ & $28^{\circ} 34^{\prime}$ & 550 \\
WYJX & Wuyuan, Jiangxi & 10 & $117^{\circ} 53^{\prime}$ & $30^{\circ} 02^{\prime}$ & 831 \\
CB & Chengbu, Hunan & 10 & $110^{\circ} 15^{\prime}$ & $26^{\circ} 20^{\prime}$ & 610 \\
KH & Kaihua, Zhejiang & 10 & $118^{\circ} 17^{\prime}$ & $29^{\circ} 14^{\prime}$ & 1453 \\
WYGD & Wengyuan, Guangdong & 10 & $114^{\circ} 04^{\prime}$ & $24^{\circ} 28^{\prime}$ & 215 \\
FN & Funing, Yunnan & 11 & $105^{\circ} 21^{\prime}$ & $23^{\circ} 30^{\prime}$ & 1100 \\
FD & Fengdu, Chongqing & 10 & $107^{\circ} 38^{\prime}$ & $29^{\circ} 48^{\prime}$ & 560 \\
JO & Jianou, Fujian & 10 & $118^{\circ} 28^{\prime}$ & $26^{\circ} 59^{\prime}$ & 204 \\
ZS & Zhoushan, Zhejiang & 10 & $121^{\circ} 60^{\prime}$ & $30^{\circ} 12^{\prime}$ & 350 \\
SZHN & Sangzhi, Hunan & 10 & $110^{\circ} 19^{\prime}$ & $29^{\circ} 42^{\prime}$ & 700 \\
SZHB & Songzi, Hubei & 10 & $111^{\circ} 46^{\prime}$ & $30^{\circ} 10^{\prime}$ & 424 \\
NJ & Nanjing, Jiangsu & 10 & $118^{\circ} 46^{\prime}$ & $31^{\circ} 51^{\prime}$ & 28 \\
GY & Guangyuan, Sichuan & 10 & $105^{\circ} 36^{\prime}$ & $32^{\circ} 18^{\prime}$ & 890 \\
CX & Cenxi, Guangxi & 10 & $111^{\circ} 10^{\prime}$ & $22^{\circ} 40^{\prime}$ & 230 \\
HuoS & Huoshan, Anhui & 10 & $116^{\circ} 15^{\prime}$ & $31^{\circ} 12^{\prime}$ & 150 \\
SC & Shangcheng, Henan & 10 & $115^{\circ} 32^{\prime}$ & $31^{\circ} 44^{\prime}$ & 410 \\
TB & Tongbai, Henan & 10 & $113^{\circ} 21^{\prime}$ & $32^{\circ} 21^{\prime}$ & 300 \\
HA & Hongan, Hubei & 10 & $114^{\circ} 38^{\prime}$ & $31^{\circ} 32^{\prime}$ & 300 \\
\hline
\end{tabular}




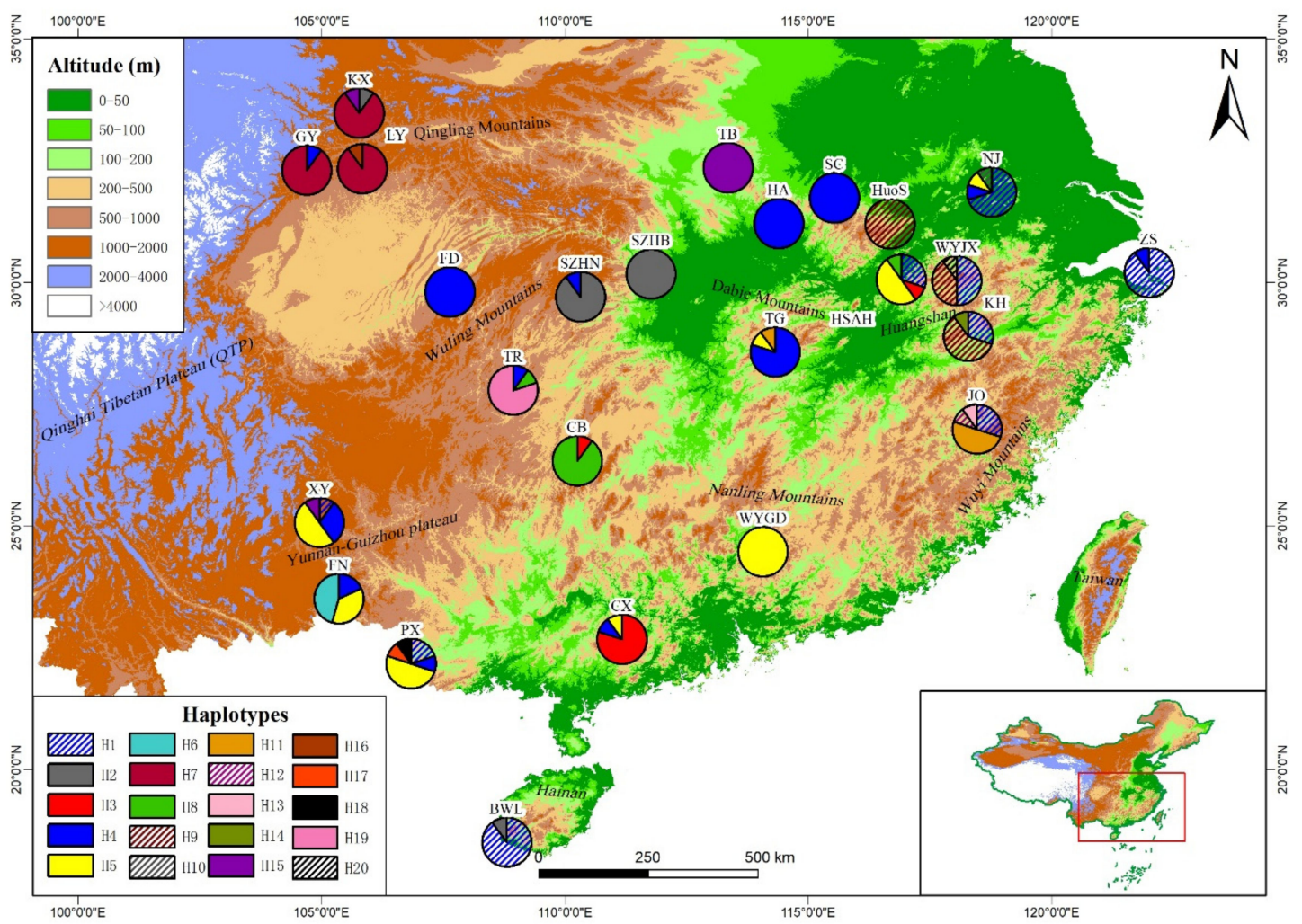

Figure 1. Geographic distribution of haplotypes (H1-H20) detected in Liquidambar formosana..

\subsection{Chloroplast DNA Amplification, Sequencing, and Sequence Alignment}

After screening variation in ten noncoding cpDNA fragments ( $p s b J-p e t A, p s b D$-trnT, ndhF-rpl32, trnL-trnF, rpl16, psbA-trnH, trnS-trnG, rpl32-trnL, petL-psbE, trnQ-rps16), four cpDNAs were used for analyses of genetic variation within and among populations of L. formosana: $\operatorname{trnL}-\operatorname{trnF}, n d h F-r p l 32$, $p s b D$-trnT, and psbJ-pet $A[24,25]$. Polymerase chain reactions (PCR) amplifications were performed in a $25 \mu \mathrm{L}$ reaction volumes containing: $12.5 \mu \mathrm{L}$ of $2 \times$ Taq buffer (Aid Lab, Beijing, China), 5 pmol of each primer, approximately $50 \mathrm{ng}$ template DNA, sterile double distilled water filled to $25 \mu \mathrm{L}$. The PCR amplification procedure for $t r n L-t r n F$ fragment included first denaturation at $94{ }^{\circ} \mathrm{C}$ for $5 \mathrm{~min}$, followed by 35 cycles of denaturation for $30 \mathrm{~s}$ at $94^{\circ} \mathrm{C}$, annealing for $30 \mathrm{~s}$ at $55^{\circ} \mathrm{C}$, and then extension for $60 \mathrm{~s}$ at $72{ }^{\circ} \mathrm{C}$, with a final extension at $72^{\circ} \mathrm{C}$ for $10 \mathrm{~min}$. For the latter three fragments, the procedure for PCR followed by the "slow and cold" of Shaw et al. [25]. PCR products were purified with a purification kit (Majorbio, Shanghai, China) and then sequenced on an ABI 3730XL DNA analyzer (Applied Biosystems, Foster, CA, USA). Regions of ambiguous alignment and incomplete data were removed from the analyses, and lacking sequences were coded as missing. All four sequences were concatenated, and then edited and aligned using Contig Express from a component of Vector NTI Suite 6.0 (Informax Inc., North Bethesda, MD, USA) and BioEdit software version 7.0.5.2 [26] with manual correction. Indels were regarded as substitutions following Caicedo and Schaal [27]. All four chloroplast haplotype sequences of $L$. formosana have been deposited in GenBank: Accession numbers MH485141-MH485147 for $p s b D-t r n T$, MH485148-MH485155 for $p s b J-p e t A$, MH485156-MH485160 for trnL-trnF, and MH485137-MH485140 for ndhF-rpl32.

\subsection{Genetic Diversity, Haplotype Network Construction, and Haplotype Distribution}

Population genetic diversity based on the four cpDNA fragments was qualified using indices of nucleotide diversity $(\pi)$, haplotype diversity $(\mathrm{Hd})$, genetic differentiation coefficient $(\mathrm{Fst})$, gene flow $(\mathrm{Nm}=((1-\mathrm{Fst})-1) / 2)$, and the number of haplotypes was calculated with the program DnaSPv5 [28]. 
The program PERMUT [29] was used to test the phylogeographic structure by comparing whether Nst was significantly greater than Gst. A significantly higher Nst than Gst indicates the presence of phylogeographical structure with relatively closer haplotypes frequently found in the same area. Nst is a parameter of genetic differentiation that takes into account distances among haplotypes, whereas Gst is a parameter of population differentiation that does not consider distances among haplotypes. Gst and Nst were estimated with 1000 permutations. Meanwhile, the total gene diversity (Ht) and average gene diversity within populations (Hs) was calculated. Relationships among chloroplast haplotypes were inferred by constructing a median-joining network with the default settings in NETWORK 5.0 [30]. The distributions of all haplotypes were plotted on a relief map of China. Several clades identified in the chloroplast network were also mapped.

\subsection{Phylogenetic Analyses and Divergence Time Estimation}

The phylogenetic tree was reconstructed by maximum likelihood (ML) and maximum parsimony (MP) using PAUP Version 4.0*b10 [31], and Bayesian analysis using MrBayes Version 3.1.2 [32], respectively. The sequences of L. styraciflua was treated as outgroup. The ML and MP analyses were performed by heuristic search methods with tree bisection reconnection (TBR) branch swapping, and a bootstrap test was conducted 1000 times. The Bayesian analysis was performed using the best-fit GTR+I model of nucleotide substitution, which was chosen from MrModeltest 2.3 [33] under the Akaike information criterion (AIC). Markov Chain Monte Carlo (MCMC) was run for 10,000,000 generations, sampling every 1000 generation. A total of 10,000 trees were sampled. The first 2500 trees were burned in, and the rest were used to reconstruct $50 \%$ consensus tree and computed posterior probabilities. The result of the tree was edited and visualized using FIGTREE v1.4.0 [34].

The divergence time of all haplotypes was estimated by BEAST 1.7.4 [35], with the uncorrelated lognormal (UCLN) relaxed-clock model. The estimated divergence time of L. styraciflua and L. formosana was $22.16 \mathrm{Ma}$ (95\% HPD (Highest posterior density): 9.74, 44.59) according to Morris et al. [36] which was used to calibrate the root node. The constant size coalescent was adopted as the tree prior, with $L$. styraciflua as outgroup. Five independent runs of $80,000,000$ MCMC generations, were conducted, sampling at every 8000 generations, following a burn in of the first $10 \%$ cycles. The log files were checked in TRACER v.1.6 [37] to confirm effective sample size (ESS) for all parameters reached adequacy (ESS $>200$ ) and convergence of the chains to a stationary distribution. Divergence time and 95\% highest posterior density (HPD) were visualized using FIGTREE v1.4.0 [34].

\subsection{Population Genetic Structure}

To study genetic structure, an analysis of molecular variance (AMOVA) was employed using the software ARLEQUIN V3.5 [38] by performing 1000 random permutations to ensure the accuracy of the estimation of variance components.

\subsection{Tests of Expansion}

To test the likelihood of departures from neutrality and evidence of range expansion, Tajima's D and Fu's Fs statistics were calculated using DnaSPv5 [28]. Significant negative values of Tajima's D and Fu's Fs may be the result of population expansion. Because the value of Fu's, Fs was more sensitive than Tajima's D, it is often used as the test parameter at present [39]. In addition, the mismatch distribution was calculated to further investigate demographic history. A multimodal distribution usually suggests that populations are at equilibrium, whereas a unimodal distribution shows that populations have experienced recent expansion. Coded indels were excluded from all tests of expansion.

\subsection{Ecological Niche Modeling}

A Biomod2 species distribution model was used to reconstruct geographic distribution patterns of L. formosana in different historical periods, including the potential distribution areas of the present (1950-2000) and the past (the last glacial maximum period (LGM: 21,000 years before present), and the 
last interglacial period (LIG: 130,000 years before present). Current distribution records were obtained from the Chinese Virtual Herbarium (http://www.cvh.org.cn/), Global Biodiversity Information Facility (https://www.gbif.org/), and the 25 records in this study. A total of 215 presence records of L. formosana were obtained from throughout the species distribution range (Figure S1). Nineteen bioclimatic variables and altitude were downloaded from the WorldClim website with a resolution of 2.5 arcmin resolution (http://www.worldclim.org/). To avoid model over-fitting and inaccurate distribution prediction, pairwise Pearson correlation coefficients $(r>0.8)$ were eliminated [40]. Only eight environmental variables with $r<0.7$ were retained for SDMs (BIO2: Mean diurnal range, BIO5: Max temperature of warmest month, BIO6: Min temperature of coldest month, BIO8: Mean temperature of wettest quarter, BIO12: Annual precipitation, BIO15: Precipitation seasonality, BIO17: Precipitation of driest quarter and BIO18: Precipitation of warmest quarter) were used to reconstruct the geographic distribution of L. formosana. We evaluated model performance using the true skill statistics (TSS), the area under the receiver operating characteristic curve (AUC) and Kappa value calculated by Biomod2.

\section{Results}

\subsection{Chloroplast Haplotype Variation, Haplotype Network, and Distribution}

The total aligned length of the four cpDNA regions was $2732 \mathrm{bp}$ (807 bp for $p s b D$-trnT, $714 \mathrm{bp}$ for $p s b J-p e t A, 747 \mathrm{bp}$ for $\operatorname{trnL}-t r n F$, and $464 \mathrm{bp}$ for $n d h F-r p l 32)$. Based on the concatenated sequences, twenty variable sites were detected, including ten nucleotide substitutions and ten indels (Supplementary Table S1). Sequences have been deposited in GenBank (accession numbers provided in Table S2). Twenty haplotypes ( $\mathrm{H} 1-\mathrm{H} 20)$ were recovered from the 25 populations, with 18 of these populations exhibiting more than one haplotype, including one population (PX) with five haplotypes, four populations (XY, HSAH, JO, and NJ) with four haplotypes each, seven populations (TR, KX, TG, WYJX, KH, FN, and CX) with three haplotypes each, and six populations (BWL, LY, CB, ZS, SZHN, and GY) with two haplotypes each, while the remaining seven populations (WYGD, FD, SZHB, HuoS, SC, TB, and HA) were fixed for a signal haplotype. Haplotypes in each population of L. formosana are listed in Table 2.

Table 2. Distribution and polymorphism of haplotypes in each population of L. formosana.

\begin{tabular}{cccc}
\hline Population & Haplotype (Number) & Hd & $\pi$ \\
\hline BWL & H1(9), H2(1) & 0.20000 & 0.00053 \\
PX & H1(2), H4(1), H5(5), H17(1), H18(1) & 0.75556 & 0.00085 \\
TR & H4(1), H8(1), H19(8) & 0.37778 & 0.00029 \\
XY & H1(1), H4(3), H5(5), H15(1) & 0.71111 & 0.00081 \\
HSAH & H1(3), H3(1), H5(5), H8(1) & 0.71111 & 0.00109 \\
KX & H2(1), H7(8), H15(1) & 0.37778 & 0.00051 \\
LY & H7(9), H16(1) & 0.20000 & 0.00015 \\
TG & H4(8), H5(1), H11(1) & 0.37778 & 0.00042 \\
WYJX & H1(5), H9(4), H20(1) & 0.64444 & 0.00041 \\
CB & H3(1), H8(9) & 0.20000 & 0.00038 \\
KH & H1(3), H9(6), H14(1) & 0.60000 & 0.00101 \\
WYGD & H5(10) & 0.00000 & 0.00000 \\
FN & H4(2), H5(4), H6(5) & 0.69091 & 0.00059 \\
FD & H4(10) & 0.00000 & 0.00000 \\
JO & H1(3), H11(5), H12(1), H13(1) & 0.71111 & 0.00120 \\
ZS & H1(9), H4(1) & 0.20000 & 0.00046 \\
SZHN & H2(9), H4(1) & 0.20000 & 0.00008 \\
SZHB & H2(10) & 0.00000 & 0.00000 \\
NJ & H1(7), H4(1), H5(1), H10(1) & 0.53333 & 0.00088 \\
GY & H4(1), H7(9) & 0.20000 & 0.00008 \\
CX & H3(8), H4(1), H5(1) & 0.37778 & 0.00029 \\
HuoS & H9(10) & 0.00000 & 0.00000 \\
SC & H4(10) & 0.00000 & 0.00000 \\
TB & H15(10) & 0.00000 & 0.00000 \\
HA & H4(10) & 0.00000 & 0.00000 \\
Total & 20(10) & 0.88762 & 0.00144 \\
\hline
\end{tabular}

Note: () in bold indicates private haplotype. 
According to the phylogenetic consensus tree, five clades could be identified by 10 nucleotide substitutions and 10 indels mutations in the haplotype network (Figure 2). The solid line indicates a nucleotide mutation step interlinking two haplotypes, and the dotted line indicates an indel mutation step. Each line indicates one mutation step. Clade I including H1, H12, H20, H9, and H10 occurred mainly in Eastern China, with the exception of BWLpopulation, each showed one or more indels mutation. Clade II contained H11 and H13, six individuals of JO and one individual of TG. Clade III containing H8, H16, and H17 detected in 13 individuals, nine individuals of CB, and the rest, four individuals, distributed in four populations. Clade IV contained only H14 and H15. The remaining haplotypes were mostly distributed in Southwestern China, Central China, Qinling and Dabieshan mountains regions, and formed Clade V (Figure 2). H4 was the most widespread haplotype, found in $19.9 \%$ of the individuals and $40 \%$ of the populations. The second (H1) and third (H5) most frequent haplotypes were detected in $16.7 \%$ and $12.7 \%$ of the individuals, respectively. $\mathrm{H} 4$ and $\mathrm{H} 1$ were internal to most of the other haplotypes. Obviously, $\mathrm{H} 5$ was an ancestral haplotype because it was scattered in geographically remote populations (XY, PX, and FN in Southwestern China, and CX, WYGD, and TG in Central China, HSAH and NJ in Eastern China) and located at a center position in the network. In addition, 11 haplotypes (H6, H10, H12, H13, H14, H16, H17, H18, H19, and H20) were private to a single population.

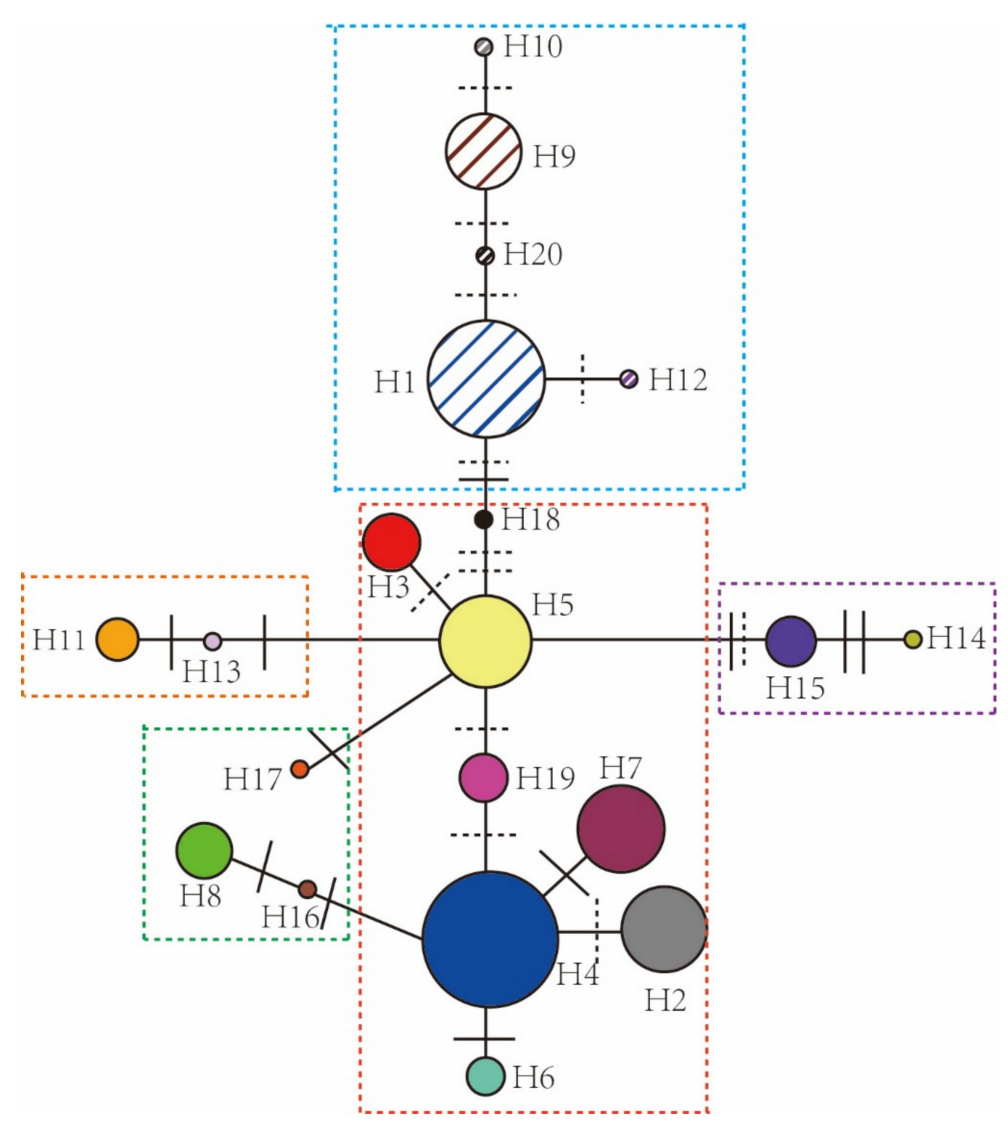

Figure 2. Network of cpDNA haplotypes detected in L. formosana. Note: The sizes of circles are proportional to the individual number of haplotypes, with the smallest circle representing $n=1$, while the largest represents $n=50$. The solid line indicates a nucleotide mutation step interlinking two haplotypes, and the dotted line indicates an indel mutation step. Each line indicates one mutation step.

\subsection{Genetic Diversity and Genetic Structure}

Total cpDNA haplotype diversity was considerably high in L. formosana $(\mathrm{Ht}=0.909 \pm 0.0192$ ). Nevertheless, diversity within populations was relatively low ( $\mathrm{Hs}=0.323 \pm 0.0553)$. The haplotype diversity $(\mathrm{Hd})$ and nucleotide diversity $(\pi)$ were 0.88762 and 0.00144 , respectively. The population 
haplotype diversity ranged from 0 to 0.75556 , and nucleotide diversity $(\pi)$ ranged from 0 to 0.00120 (Table 2). Permutation test showed that Nst (0.730) was significantly higher than Gst $(0.645 ; p<0.05)$, indicating significant phylogeographical structure across the whole natural range of L. formosana in China. Analysis of molecular variance (AMOVA) (Table 3) indicated that a large proportion (75.34\%) of the chloroplast variation in L. formosana was resided between populations, whereas the component of genetic variation within populations was only $24.66 \%$. The variation of chloroplast variation between populations had reached a significant level (Fst $=0.75, p<0.01)$.

Table 3. Analysis of molecular variance (AMOVA) of cpDNA data of L. formosana populations.

\begin{tabular}{ccccc}
\hline Source of Variation & $\begin{array}{c}\text { Degree of } \\
\text { Freedom }\end{array}$ & $\begin{array}{c}\text { Sum of } \\
\text { Squares }\end{array}$ & $\begin{array}{c}\text { Variance } \\
\text { Components }\end{array}$ & $\begin{array}{c}\text { Percentage of } \\
\text { Variation (\%) }\end{array}$ \\
\hline Among populations & 24 & 923.722 & $3.71250 \mathrm{Va}$ & 75.34 \\
Within populations & 226 & 274.700 & $1.21549 \mathrm{Vb}$ & 24.66 \\
Total & 250 & 1198.422 & 4.92799 & Fst $=0.75$ \\
\hline
\end{tabular}

\subsection{Population History Dynamic Analysis and Divergence Time Estimation}

The value of Tajima's D $(-0.87277, p>0.10)$ was not significantly negative. However, the value of Fu's Fs was significantly negative $(-2.668, p<0.05)$ for these four concatenated sequences. Significant negative values of Fu's Fs may be the result of population expansion. To further investigate the demographic history, mismatch distribution analysis was also plotted (Figure 3). Deviation of observed values from those expected and a strongly unimodal mismatch distribution suggested the sudden spatial or demographic expansion of L. formosana. Neutrality test and the mismatch distribution analysis showed L. formosana experienced an expansion event in history.

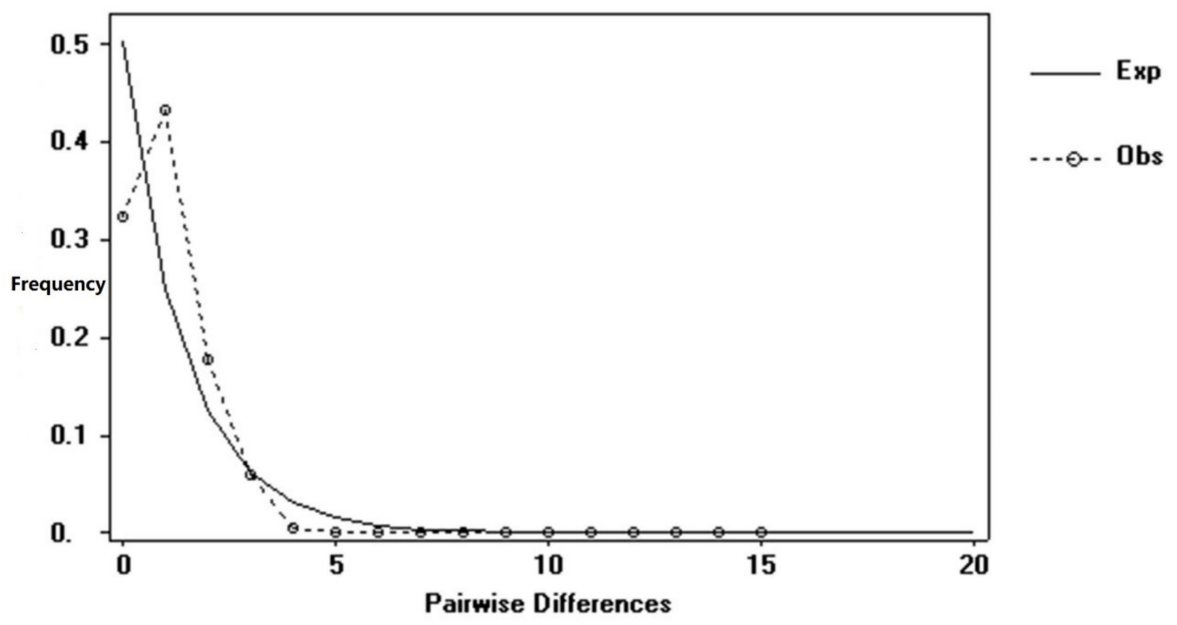

Figure 3. Mismatch distribution analysis of cpDNA haplotypes for L. formosana populations. The vertical axis represents frequency, whereas the horizontal axis shows the number of pairwise differences. Note: The line represents the distributions expected for a population, and the dotted line represents the observed distribution.

The divergence time BEAST analysis suggests that the time of the most recent common ancestor for clade V mostly distributed in Southwestern China, Central China, Qinling and Dabieshan mountains regions was dated to $10.30 \mathrm{Ma}$ (95\% HPD: 9.74-15.28), belonging to the middle Miocene period. The divergence time of clade I occurred mainly in Eastern China was 7.65 Ma (95\% HPD: 2.49-12.15), belonging to the late Miocene period. Clade II and clade IV were all derived from the ancient haplotype H5, and the divergence time was 1.13 Ma (95\% HPD: 0.02-4.29) and 2.10 Ma (95\%HPD: 0.10-6.34), respectively. Clade I and clade III were relatively special, including 2 haplotypes derived from a wide distribution of $\mathrm{H} 4$ and a private haplotype derived from ancestral haplotype $\mathrm{H} 5$, the divergence 
time was 1.50 MYA (95\% HPD: 0.07-4.91) (Figures 2 and 4). The divergence time of the latter three groups belongs to the Quaternary period. Five clades could be clearly identified to the phylogenetic relationships of haplotype detected in the BEAST analysis, which had high probability values and correspond closely to the haplotype network (Figure 2).

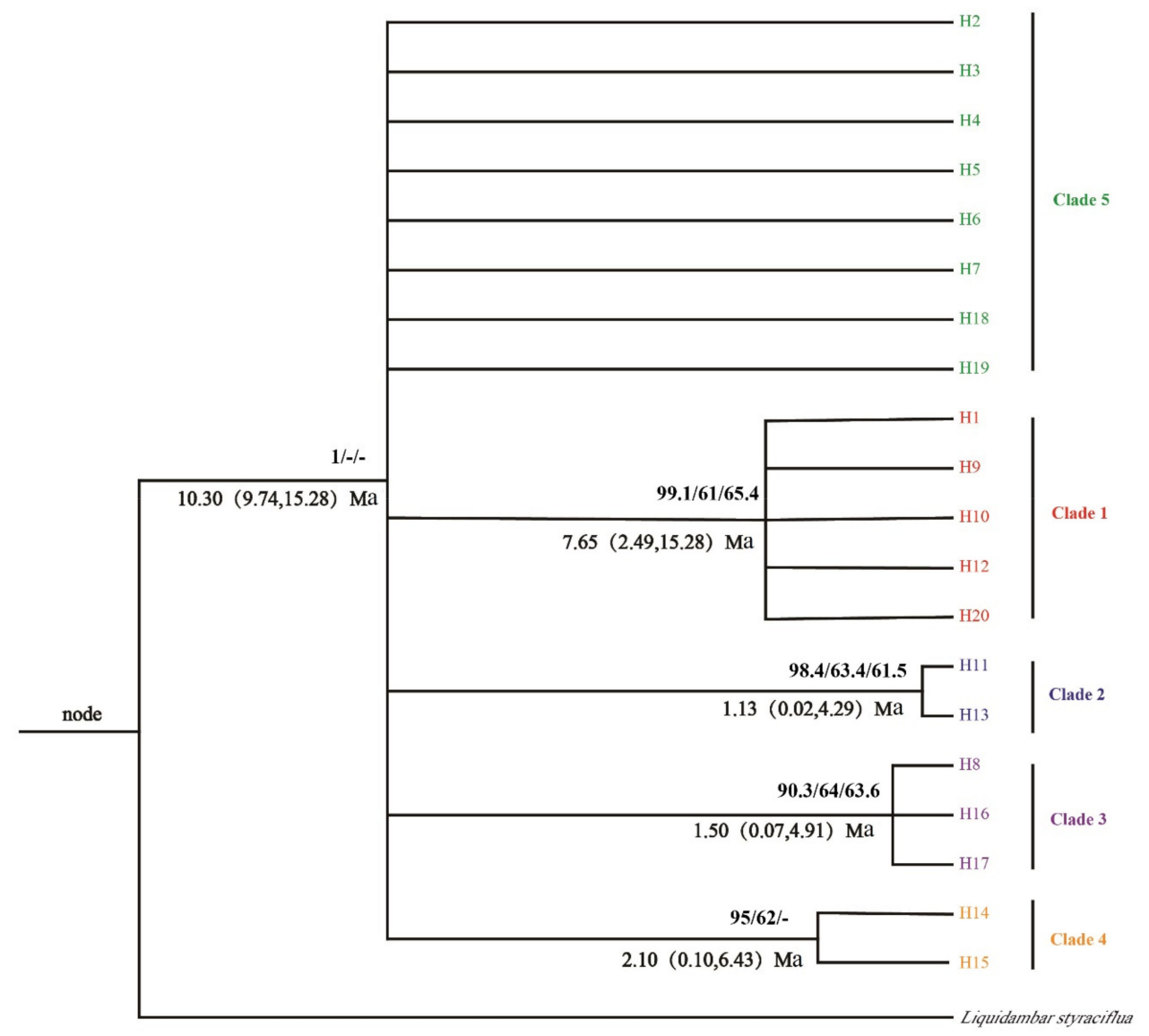

Figure 4. Phylogenetic tree of L. formosana based on 20 haplotypes result from combined analysis of four cpDNA sequence data, the values on the branches are the posterior probability $(>0.9)$ and bootstrap support values (>50\%) for the maximum-likelihood (ML) and maximum-parsimony (MP) analyses; divergence time and 95\% HPD are given (in millions of years) under the branches.

\subsection{Ecological Niche Model}

The Biomod2 species distribution model (SDM) performance was assessed using three different statistics: the true skill statistics (TSS), the area under the receiver operating characteristic curve (AUC), and Kappa value. All models performed well (TSS = 0.940, ranged from 0.772 to 0.992; AUC = 0.975, ranged from 0.886 to 0.996 ; Kappa $=0.937$, ranged from 0.772 to 0.968 ), which indicated good predictive performance (Figure S2). Based on the potential distributions of the present (1950-2000) of L. formosana, it is widely distributed across three climatic zones: tropical, subtropical, and south temperate regions. North form Qinling and Dabieshan Mountains, south to Hainan, west to Sichuan and Guizhou, and east to Taiwan $\left(18^{\circ}-34^{\circ} \mathrm{N}, 102.1^{\circ}-122.7^{\circ} \mathrm{E}\right)$. The predicted distribution under the present was consistent with their existing distribution, indicating that the distribution had reached its maximum range. The predicted distributions are actually almost identical in the present, LGM (21 kya), and LIG (130 kya), except for some peripheral areas where the concentration is concentrated on the edge of the Tibetan area and southern Yunnan and Taiwan regions. Along the distribution of the north rim, especially, the probability of occurrence is very low, and we probably should not draw many conclusions from these areas (Figure 5). The model results suggest no contraction-expansion dynamics. Instead, this was the overwhelming signal of range stability through time. 

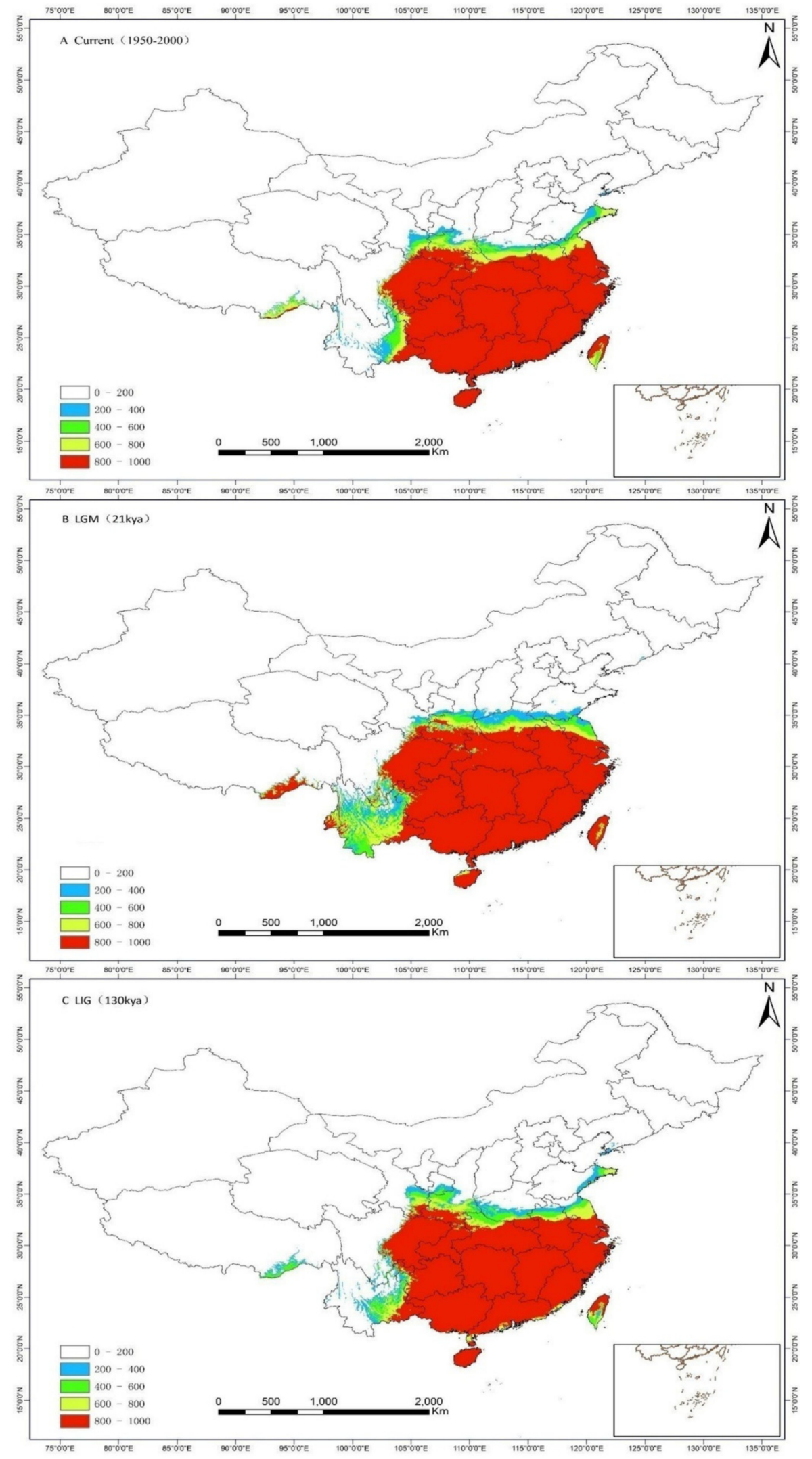

Figure 5. Maps of potential distributions as a probability of occurrence for L. formosana in China at different times through Biomod2 species distribution model using eight bioclimatic variables. (A), at present (1950-2000); (B), LGM (21 kya); (C), LIG (130 kya). Predicted distribution probabilities are shown in the legend, the deeper color representing the greater the probability distribution. 


\section{Discussion}

\subsection{Genetic Diversity and Genetic Differentiation of cpDNA Haplotype in L. Formosana}

The total cpDNA haplotype diversity $(\mathrm{Ht}=0.909)$ detected in our study was considerably higher than the average value $(\mathrm{Ht}=0.67)$ of 170 species using cpDNA markers reported by Petit [41]. These may be attributed to a long evolutionary history of the tertiary relict plant, which could accumulate more nucleotide mutations [42]. Relatively high chloroplast haplotype diversity was also found in some relict plants, such as Alsophila spinulosa $(\mathrm{Ht}=0.93)[43]$, Dipentodon sinicus $(\mathrm{Ht}=0.90)$ [44], and Tetracentron sinense $(\mathrm{Ht}=0.93)$ [45]. Widespread species or/and hotspots species may be possible explanations for the high chloroplast haplotype diversity. In general, widespread species had higher genetic variation than those of narrowly distributed species, due to wider geographical distribution providing more opportunities for gene isolation, genetic drift, and gene mutation [46].

The higher cpDNA chloroplast genetic variation was inconsistent with the moderate SSR genetic variation reported by a previous study [18]. This may have been caused by pollen and seed mediating gene flow. Nuclear genes, such as SSR markers belonging to the biparental inheritance, reflect the gene flow mediating both pollen and seed. However, cpDNA is maternal inheritance in most plants which reflect part of gene flow only, including seed mediating. The limited seed mediating gene flow of cpDNA markers weakens the homogeneity among populations which might retain the high chloroplast genetic variation. The pattern of seed dispersion could have a significant impact on the genetic differentiation of chloroplasts in the population [47]. Generally speaking, the degree of genetic differentiation of a population is negatively correlated with the transmission distance of seeds. The population differentiation due to seed transmitted by gravity is greater than by wind. Sweetgum is a wind-pollinated species, and seed-dispersing mainly by gravity. In the previous study of L. styraciflua, instead of individually dispersing seeds, many whole sweetgum fruits (each containing up to 50 seeds) were directly located around the seed tree (50 m-100 m) [48].

Total cpDNA haplotype diversity was high $(\mathrm{Ht}=0.909)$ in L. formosana. Nevertheless, diversity within populations was relatively low $(\mathrm{Hs}=0.323)$, probably caused by restricted gene flow. This was also consistent with the lower gene flow $(\mathrm{Nm}=0.18)$ detected in our study. High haplotype diversity may reflect the wide distribution of L. formosana in subtropical China, which had a large and stable population size. Compared with other species of Liquidambar distributed in North America and Turkey (e.g., L. styraciflua [36] and L. orientalis [49]), which experienced a dramatic reduction in the distribution area, L. formosana had no contraction-expansion dynamics and a stable range.

\subsection{Phylogeographic Structure and Inference of Demographic History}

The populations of L. formosana in China had a significant phylogeographical structure (Nst $=0.730$ $>$ Gst $=0.645 ; p<0.05$ ). However, the mantel test (not listed) showed there was no geographic isolation among populations, indicating that geographical distance was not the main factor causing genetic variation of L. formosana. High genetic differentiation was not completely caused by distance isolation but by natural barriers, such as mountains and rivers [50]. Therefore, another cause for the phylogeographical structure of L. formosana may be the complex topography. For example, extending from east to west are the Qinling and Dabashan mountain and north to south the Dabie mountains. Due to the blocking of the mountains, only limited genes can flow among populations. Fu's Fs value and the mismatch distribution analyses indicated there was population expansion detected in L. formosana (Figure 3). However, based on the result of SDM, no contraction-expansion dynamics were detected since LIG (130 kya). Therefore, we speculated the expansion time may happen before the period of LIG.

Using the uncorrelated lognormal relaxed-clock model, the divergence time (10.30 Ma; 95\% HPD, 9.74-15.28 Ma; Figure 4) of clade V haplotypes and 7.65 Ma (95\% HPD: 2.49-12.15) of clade I for L. formosana was an ancient origin of genetic structure, belonging to the middle and late Miocene period. The result indicated that the earliest lead to genetic differentiation was due to the Neogene geology and climate events, as confirmed by the fossil pollen records from Liquidambar. Fossils of 
Liquidambar fruit, pollen, and leaves have been commonly found in Mid-Cretaceous and Neogene of the Northern Hemisphere [51], and L. formosana has a long fossil record of pollen (about $70 \mathrm{Ma}$ ) in the northeast of China [52]. In addition, it maintains a stable range and even increases effective population size through time which is suggested by history dynamic analysis and SDM results (Figures 3 and 5) and has a wide geographical distribution and ecological amplitude which allows it to retain ancient origin [53]. The significant uplift of the Qinghai-Tibet Plateau during the middle and late Miocene (approximately 8-10 Ma) and the intensified Asian monsoons (approximately 8-9 Ma) were regarded as the two main factors driving diversification of plants during Miocene in China [54,55]. Evidence supporting middle and late Miocene diversification of other subtropical woody species has been found in phylogeographic studies, e.g., Ulmus lamellosa 9.27 Ma (5.17-13.33) [56], Tetracentron sinense 9.6 Ma (2.2-27.0) [45], Quercus glauca 9.07 Ma (5.16-13.32) [57]. The divergence time of haplotypes between H11 and H13; H8, H16, and H17; H14 and H15 were 1.13 Ma (95\%HPD: 0.02-4.29), 2.10 Ma (95\% HPD: 0.10-6.34), and 1.50 Ma (95\% HPD: 0.07-4.91) (Figure 4), respectively. Thus, we speculated that Quaternary climatic oscillations and associated series of environmental changes had also caused the diversification within L. formosana, and they are relatively complicated.

\section{Conclusions}

In summary, dramatic changes since the Miocene and associated climate cooling changes may have driven the ancestors of L. formosana to retreat from the high latitudes of the Northern Hemisphere to subtropical China in which the establishment and initial intensification of the Asian monsoon provided conditions for their ecological requirements. This scenario was confirmed by the fossil record. We revealed the ancient origin dating back to the middle Miocene (10.30 Ma; 95\% HPD, 9.74-15.28 Ma) of L. formosana. SDM results indicated there was no contraction-expansion dynamics and a stable range since the LIG. Compared with the population expansion detected by Fu's Fs value and the mismatch distribution, we speculated the expansion time might happen before LIG.

Supplementary Materials: The following are available online at http:/www.mdpi.com/1999-4907/10/10/858/s1. Table S1: Variable sites in the alignment of 20 cpDNA haplotypes of L. formosana in China; Table S2: GenBank accession numbers for 20 cpDNA haplotypes of L. formosana in China; Figure S1: The geographic distribution of L. formosana in China; Figure S2: TSS values (A), ACU values (B), and KAPPA values (C) for the ten models in predicting the distribution areas of L. formosana.

Author Contributions: R.S., F.L. and Y.Z. conceived and designed the experiments; R.S. and F.L. collected plant materials; R.S. performed the experiments and drafted the manuscript; F.L., Y.Z., P.H., X.Y. and J.L. revised the manuscript. All authors read and approved the manuscript.

Funding: This research was funded by the project of Special Funds from Laboratory of Forest Silviculture and Tree Cultivation (No. ZDRIF201713) and the Gene Discovery and Innovative Use of Forest Genetic Resources (2013BAD01B06) and the National Forest Genetic Resource Platform (2005DKA21003).

Acknowledgments: We are grateful for the people who helped us in the field observation. Especially, thank Jinchang Yang, Ru Liu, Hongdong Pang, and Chaoyi Deng for their help in sample collection.

Conflicts of Interest: The authors declare no conflict of interest.

\section{References}

1. Zachos, J.; Pagani, M.; Sloan, L.; Thomas, E.; Billups, K. Trends, rhythms, and aberrations in global climate $65 \mathrm{Ma}$ to present. Science 2001, 292, 686-693. [CrossRef] [PubMed]

2. Guo, Z.T.; Sun, B.; Zhang, Z.S.; Peng, S.Z.; Xiao, G.Q.; Ge, J.Y.; Hao, Q.Z.; Qiao, Y.S.; Liang, M.Y.; Liu, J.F.; et al. A major reorganization of Asian climate by the early Miocene. Clim. Past 2008, 4, 153-174. [CrossRef]

3. Clift, P.; Lee, J.I.; Clark, M.K.; Blusztajn, J. Erosional response of South China to arc rifting and monsoonal strengthening; a record from the South China Sea. Mar. Geol. 2002, 184, 207-226. [CrossRef]

4. Guo, Z.T.; Ruddiman, W.F.; Hao, Q.Z.; Wu, H.B.; Qiao, Y.S.; Zhu, R.X.; Peng, S.Z.; Wei, J.J.; Yuan, B.Y.; Liu, T.S. Onset of Asian desertification by $22 \mathrm{Myr}$ ago inferred from loess deposits in China. Nature 2002, 416, 159. [CrossRef] 
5. Klootwijk, C.T.; Gee, J.S.; Peirce, J.W.; Smith, G.M.; Mcfadden, P.L. An early India-Asia contact: Paleomagnetic constraints from Ninetyeast Ridge, ODP Leg 121. Geology 1992, 20, 395. [CrossRef]

6. Wan, S.; Li, A.; Clift, P.D.; Stuut, J.B.W. Development of the East Asian monsoon: Mineralogical and sedimentologic records in the northern South China Sea since 20Ma. Palaeogeogr. Palaeoclimatol. Palaeoecol. 2007, 254, 561-582. [CrossRef]

7. An, Z.S.; Kutzbach, J.E.; Prell, W.L.; Porter, S.C. Evolution of Asian monsoons and phased uplift of Himalaya-Tibetan plateau since Late Miocene times. Nature 2001, 411, 62-66.

8. Chou, Y.W.; Wang, C.N. Refugia and phylogeography of Taiwania in East Asia. J. Biogeogr. 2011, 38, 1992-2005. [CrossRef]

9. Kou, Y.; Cheng, S.; Shuang, T.; Bo, L.; Zhang, Z. The antiquity of Cyclocarya paliurus (Juglandaceae) provides new insights into the evolution of relict plants in subtropical China since the late Early Miocene. J. Biogeogr. 2016, 43, 351-360. [CrossRef]

10. Wu, Z.Y. Vegetation of China; Science Press: Beijing, China, 1980.

11. Tian, S.; Lei, S.Q.; Hu, W.; Deng, L.L.; Li, B.; Meng, Q.L.; Soltis, D.E.; Soltis, P.S.; Fan, D.M.; Zhang, Z.Y. Repeated range expansions and inter-/postglacial recolonization routes of Sargentodoxa cuneata (Oliv.) Rehd. et Wils. (Lardizabalaceae) in subtropical China revealed by chloroplast phylogeography. Mol. Phylogenet. Evol. 2015, 85, 238-246. [CrossRef]

12. Chen, J.; Hao, Z.; Xu, H.; Yang, L.; Liu, G.; Sheng, Y.; Zheng, C.; Zheng, W.; Cheng, T.; Shi, J. The complete chloroplast genome sequence of the relict woody plant Metasequoia glyptostroboides Hu et Cheng. Front. Plant Sci. 2015, 6, 447. [CrossRef] [PubMed]

13. Qi, X.S.; Chen, C.; Comes, H.P.; Sakaguchi, S.; Liu, Y.H.; Tanaka, N.; Sakio, H.; Qiu, Y.X. Molecular data and ecological niche modelling reveal a highly dynamic evolutionary history of the East Asian Tertiary relict Cercidiphyllum (Cercidiphyllaceae). New Phytol. 2012, 196, 617-630. [CrossRef] [PubMed]

14. Kuprianova, L.A. Palynological data contributing to the history of Liquidambar. Pollen Spores 1960, 2, 71-88.

15. The Angiosperm Phylogeny Group. An update of the Angiosperm Phylogeny Group classification for the orders and families of flowering plants AGP II. Bot. J. Linn. Soc. 2003, 141, 399-436. [CrossRef]

16. Ozturk, M.; Celik, A.; Guvensen, A.; Hamzaoğlu, E. Ecology of tertiary relict endemic Liquidambar orientalis Mill. forests. For. Ecol. Manag. 2008, 256, 510-518. [CrossRef]

17. Ickert-Bond, S.M.; Pigg, K.B.; Wen, J. Comparative infructescence morphology in Liquidambar (Altingiaceae) and its evolutionary significance. Am. J. Bot. 2005, 92, 1234-1255. [CrossRef] [PubMed]

18. Sun, R.; Lin, F.; Huang, P.; Zheng, Y. Moderate Genetic Diversity and Genetic Differentiation in the Relict Tree Liquidambar formosana Hance Revealed by Genic Simple Sequence Repeat Markers. Front. Plant Sci. 2016, 7, 1411. [CrossRef]

19. Wu, W. Natural Hybridization, Phylogeography and Speciation Patterns of Altingiaceae. Ph.D. Thesis, Sun Yat-sen University, Guangzhou, China, 2009.

20. Bi, X.Q.; Jin, X.Z.; Li, H.J. Genetic diversity in the natural populations of Liquidambar formosana revealed by ISSR molecular markers. Bull. Bot. Res. 2010, 30, 120-125.

21. Liu, L.X.; Li, R.; Worth, J.R.P.; Li, X.; Li, P.; Cameron, K.M.; Fu, C.X. The Complete Chloroplast Genome of Chinese Bayberry (Morella rubra, Myricaceae): Implications for Understanding the Evolution of Fagales. Front. Plant Sci. 2017, 8, 968. [CrossRef]

22. Wen, Z.; Zhe, X.; Zhang, H.; Ying, F. Chloroplast phylogeography of a desert shrub, Calligonum calliphysa (Calligonum, Polygonaceae), in arid Northwest China. Biochem. Syst. Ecol. 2015, 60, 56-62. [CrossRef]

23. Wolfe, K.H.; Li, W.H.; Sharp, P.M. Rates of nucleotide substitution vary greatly among plant mitochondrial, chloroplast, and nuclear DNAs. Proc. Natl. Acad. Sci. USA 1987, 84, 9054-9058. [CrossRef] [PubMed]

24. Taberlet, P.; Gielly, L.; Pautou, G.; Bouvet, J. Universal primers for amplification of three non-coding regions of chloroplast DNA. Plant Mol. Biol. 1991, 17, 1105-1109. [CrossRef] [PubMed]

25. Shaw, J.; Lickey, E.B.; Schilling, E.E.; Small, R.L. Comparison of whole chloroplast genome sequences to choose noncoding regions for phylogenetic studies in angiosperms: The tortoise and the hare III. Am. J. Bot. 2007, 94, 275-288. [CrossRef] [PubMed]

26. Hall, T.A. A User-Friendly Biological Sequence Alignment Editor and Analysis Program for Windows 95/98/NT. Nucleic Acids Symp. Ser. 1999, 41, 95-98.

27. Caicedo, A.L.; Schaal, B.A. Population structure and phylogeography of Solanum pimpinellifolium inferred from a nuclear gene. Mol. Ecol. 2004, 13, 1871-1882. [CrossRef] [PubMed] 
28. Librado, P.; Rozas, J. DnaSP v5: A software for comprehensive analysis of DNA polymorphism data. Bioinformatics 2009, 25, 1451-1452. [CrossRef] [PubMed]

29. Pons, O.; Petit, R.J. Measuring and testing genetic differentiation with ordered versus unordered alleles. Genetics 1996, 144, 1237-1245.

30. Bandelt, H.J.; Forster, P.; Rohl, A. Median-joining networks for inferring intraspecific phylogenies. Mol. Biol. Evol. 1999, 16, 37-48. [CrossRef]

31. Swofford, D.L. PAUP*: Phylogenetic Analysis Using Parsimony (and Other Methods) 4.0 Beta; Sinauer Associates: Sun-derland, MA, USA, 2002.

32. Ronquist, F.; Huelsenbeck, J.P. MrBayes 3: Bayesian phylogenetic inference under mixed models. Bioinformatics 2003, 19, 1572-1574. [CrossRef]

33. Posada, D.; Buckley, T.R. Model selection and model averaging in phylogenetics: Advantages of akaike information criterion and bayesian approaches over likelihood ratio tests. Syst. Biol. 2004, 53, 793-808. [CrossRef]

34. Rambaut, A. FigTree v1. 4.0. A Graphical Viewer of Phylogenetic Trees; Institute of Evolutionary Biology University of Edinburgh: Edinburgh, UK, 2012.

35. Drummond, A.J.; Suchard, M.A.; Xie, D.; Rambaut, A. Bayesian phylogenetics with BEAUti and the BEAST 1.7. Mol. Biol. Evol. 2012, 29, 1969-1973. [CrossRef] [PubMed]

36. Morris, A.B.; Ickert-Bond, S.M.; Brunson, D.B.; Soltis, D.E.; Soltis, P.S. Phylogeographical structure and temporal complexity in American sweetgum (Liquidambar styraciflua; Altingiaceae). Mol. Ecol. 2008, 17, 3889-3900. [CrossRef]

37. Rambaut, A.; Suchard, M.A.; Xie, D.; Drummond, A.J. Tracer v1.6. 2014. Available online: http://beast.bio.ed. ac.uk/Tracer (accessed on 31 January 2017).

38. Excoffier, L.; Lischer, H. Arlequin suite ver 3.5: A new series of programs to perform population genetics analyses under Linux and Windows. Mol. Ecol. Resour. 2010, 10, 564-567. [CrossRef] [PubMed]

39. Pilkington, M.M.; Wilder, J.A.; Mendez, F.L.; Cox, M.P.; Woerner, A.; Angui, T.; Kingan, S.; Mobasher, Z.; Batini, C.; Destro-Bisol, G.; et al. Contrasting signatures of population growth for mitochondrial DNA and Y chromosomes among human populations in Africa. Mol. Biol. Evol. 2008, 25, 517-525. [CrossRef] [PubMed]

40. Peterson, A.T.; Nakazawa, Y. Environmental data sets matter in ecological niche modeling: An example with. Glob. Ecol. Biogeogr. 2010, 17, 135-144.

41. Petit, R.J.; Duminil, J.; Fineschi, S.; Hampe, A.; Salvini, D.; Vendramin, G.G. Comparative organization of chloroplast, mitochondrial and nuclear diversity in plant populations. Mol. Ecol. 2005, 14, 689-701. [CrossRef] [PubMed]

42. Huang, S.; Chiang, Y.C.; Schaal, B.A.; Chou, C.H.; Chiang, T.Y. Organelle DNA phylogeography of Cycas taitungensis, a relict species in Taiwan. Mol. Ecol. 2001, 10, 2669-2681. [CrossRef]

43. Su, Y.; Wang, T.B.; Jiang, Y.; Chen, G.; Ouyang, P.; Sun, Y. Genetic differentiation of relictual populations of Alsophila spinulosa in southern China inferred from cpDNA trnL-F noncoding sequences. Mol. Phylogenet. Evol. 2005, 34, 323-333. [CrossRef]

44. Yuan, Q.J.; Zhang, Z.Y.; Peng, H.; Ge, S. Chloroplast phylogeography of Dipentodon (Dipentodontaceae) in southwest China and northern Vietnam. Mol. Ecol. 2008, 17, 1054-1065. [CrossRef] [PubMed]

45. Sun, Y.; Moore, M.J.; Yue, L.; Feng, T.; Chu, H.; Chen, S.; Ji, Y.; Wang, H.; Li, J. Chloroplast phylogeography of the East Asian Arcto-Tertiary relict Tetracentron sinense (Trochodendraceae). J. Biogeogr. 2014, 41, 1721-1732. [CrossRef]

46. Hamrick, J.L.; Godt, M.J.W. Effects of Life History Traits on Genetic Diversity in Plant Species. Philos. Trans. Biol. Sci. 1996, 351, 1291-1298.

47. Petit, R.J.; Itziar, A.; Jacques-Louis, D.B.; Christiane, B.; Simon, B.; Rachid, C.; Richard, E.; Silvia, F.; Delphine, G.; Martin, L. Glacial refugia: Hotspots but not melting pots of genetic diversity. Science 2003, 300, 1563-1565. [CrossRef] [PubMed]

48. Nuttle, T.; Haefner, J.W. Seed dispersal in heterogeneous environments: Bridging the gap between mechanistic dispersal and forest dynamics models. Am. Nat. 2005, 165, 336-349. [CrossRef] [PubMed]

49. Ozdilek, A.; Cengel, B.; Kandemir, G.; Tayanc, Y.; Velioglu, E.; Kaya, Z. Molecular phylogeny of relict-endemic Liquidambar orientalis Mill based on sequence diversity of the chloroplast-encoded mat K gene. Plant Syst. Evol. 2012, 298, 337-349. [CrossRef] 
50. Koizumi, I.; Yamamoto, S.; Maekawa, K. Decomposed pairwise regression analysis of genetic and geographic distances reveals a metapopulation structure of stream-dwelling Dolly Varden charr. Mol. Ecol. 2006, 15, 3175-3189. [CrossRef] [PubMed]

51. Ferguson, D.K. A survey of the Liquidambaroideae (Hamamelidaceae) with a view to elucidating its fossil record. Evol. Syst. Foss. Hist. Hamamelidae 1989, 1, 249-272.

52. Sun, X. Palynofloristical inveatgation on the late cretaceous and paleocene of China. Acta Phytotaxon. Sin. 1979, 17, 8-23.

53. Avise, J.C. Phylogeography: Retrospect and prospect. J. Biogeogr. 2010, 36, 3-15. [CrossRef]

54. Royden, L.H.; Burchfiel, B.C.; Van Der Hilst, R.D. The geological evolution of the Tibetan Plateau. Science 2008, 321, 1054-1058. [CrossRef]

55. An, Z.; Huang, Y.; Liu, W.; Guo, Z.; Clemens, S.; Li, L.; Prell, W.; Ning, Y.; Cai, Y.; Zhou, W. Multiple expansions of $\mathrm{C} 4$ plant biomass in East Asia since $7 \mathrm{Ma}$ coupled with strengthened monsoon circulation. Geology 2005, 33, 705-708.

56. Liu, L.; Chen, W.; Yan, D.T.; Li, J.; Liu, L.; Wang, Y.L. Molecular phylogeography and paleodistribution modeling of the boreal tree species Ulmus lamellosa (T. Wang et S.L. Chang) (Ulmaceae) in China. Tree Genet. Genomes 2017, 13, 11. [CrossRef]

57. Xu, J.; Deng, M.; Jiang, X.L.; Westwood, M.; Song, Y.G.; Turkington, R. Phylogeography of Quercus glauca (Fagaceae), a dominant tree of East Asian subtropical evergreen forests, based on three chloroplast DNA interspace sequences. Tree Genet. Genomes 2015, 11, 1-17. [CrossRef]

(C) 2019 by the authors. Licensee MDPI, Basel, Switzerland. This article is an open access article distributed under the terms and conditions of the Creative Commons Attribution (CC BY) license (http://creativecommons.org/licenses/by/4.0/). 